Ann. Abeille, 1965, 8 (2), 83-93.

\title{
SUR QUELQUES POLLINISATEURS DES LÉGUMINEUSES FOURRAGÈRES
}

\author{
J. LECOMTE et S. TIRGARI \\ Station de Recherches sur l'Abeille et les Insectes sociaux, \\ Bures-sur-Yvette (Seine-et-Oise)
}

SOMMAIRE

Les auteurs exposent les premiers résultats d'un inventaire des pollinisateurs des Légumineuses fourragères dans plusieurs régions de la France.

La liste des insectes observés, ainsi que des aperçus sur leur comportement et leur biologie sont donnés afin de pouvoir apprécier leur intérêt en ce qui concerne la pollinisation.

\section{INTRODUC'TION}

Ces dernières années ont été marquées par une série de travaux mettant en évidence le rôle des Apoïdes en tant qu'agents pollinisateurs de plusieurs légumineuses importantes sur le plan économique : la Luzerne (Medicago sativa L.), le Trèfle violet (Trifolium pratense L.) et le Trèfle blanc (Trifolium repens L.).

LINSLEY (I946) donne le premier inventaire des pollinisateurs de la Luzerne en Californie. Popov (I95I) établit une liste similaire en Asie centrale. BoHarT (I957-I958) passe en revue et analyse les renseignements relatifs aux pollinisateurs les plus importants des légumineuses fourragères de l'ouest et du centre des ÉtatsUnis, de l'Argentine, Australie, Europe centrale et Asie centrale. Arnason (Ig64) mentionne les résultats obtenus au Canada au cours de recherches sur la pollinisation des plantes cultivées. En Europe, Moczar ( $196 \mathbf{r}$ ) a étudié très en détail la faune des abeilles sauvages des champs de Luzerne en Hongrie. En France, Chauvin (r952-I960) a étudié la faune des champs cuitivés et spécialement celle du champ de Luzerne, mais sans prêter un intérêt particulier aux pollinisateurs. En fait, le premier travail sur la question est celui de LECOMTE (I959-62). 


\section{MÉTHODES ET DESCRIPTION DES LOCALITÉS ÉTUDIÉES}

Depuis plusieurs années et essentiellement durant les étés de 1963 et 1964 , nous avons étudié la faune pollinisatrice des légumineuses fourragères dans la région parisienne, le centre-ouest et le sud-ouest de la France. Les observations et les récoltes ont été effectuées sur les champs rencontrés le long des routes ou connus comme devant servir à la production de graines.

Des observations beaucoup plus suivies ont été effectuées à la Station d'Améliorat ion des Plantes fourragères à Lusignan (Vienne). Dans cette localité, nous disposions de trois hectares de parcelles destinées à des études de sélection ainsi que des essais de multiplication se trouvant sur d'autres parties du domaine et, naturellement, des cultures privées qui existaient au voisinage.

Le site de Lusignan s'est révélé tout particulièrement favorable à cette ét ude. En effet, sa situation géographique en fait le point de rencontre d'influences méridionales et septentrionales qui permettent à un grand nombre d'espèces de prospérer, d'aut're part, il s'agit d'une région traditionnelle de culture de luzerne à graine où l'évolution de l'agriculture n'a pas encore d'influences néfastes sur les insectes pollinisateurs ; enfin, les champs expérimentaux de la station et le fait que les différentes parcelles fleurissent à des époques très variables, assurant une continuité durant tout l'été, ont une influence certainement heureuse sur l'importance des populations.

\section{LES POLLINISATEURS, LEUR IDENTITÉ ET LEUR ABONDANCE}

Nous avons pu constater la présence de 2 I espèces d'abeilles autres que l'abeille domestique, représentant six familles d'Apoidea sur les légumineuses fourragères étudiées. Comme on pouvait s'y attendre, l'abondance et les proportions relatives des différentes espèces varient considérablement d'une localité à l'autre, d'une année à l'autre et même d'une semaine à l'autre dans un même lieu.

Dans le tableau $x$, nous donnons la liste des insectes rencontrés à Lusignan avec quelques indications sur leur abondance approximative.

En ce qui concerne les autres régions étudiées, nous n'avons rencontré qu'en deux circonstances des insectes absents de la région de Lusignan. L'un d'entre nous avait noté en I 960 dans la région d'Auneau (Eure-et-Loir) une certaine abondance de Rophites canus EV. En I963 et I964, ils étaient devenus beaucoup plus rares. En I960, également, l'un d'entre nous avait constaté la grande abondance d'Halictus longulus sM, femelle d'été d'Halictus malachurus $\mathrm{K}$. dans les champs du marais breton en Vendée.

\section{RENSEIGNEMEN'TS PARTICULIERS}

\section{AUX POLLINISATEURS RENCONTRÉS}

L'abondance seule d'un insecte sur les fleurs n'est pas un bon indice de son importance en tant que pollinisateur. L'efficacité de l'insecte est liée à bien des facteurs En particulier, en ce qui concerne les régions étudiées, la tolérance de condition; climatiques généralement défavorables à l'activité des Hyménoptères peut être considérée comme particulièrement importante.

Nous avons eu l'occasion d'effectuer un grand nombre d'observations sur le 
comportement des insectes par temps défavorable au cours de ig63 qui fut particulièrement pluvieux et anormalement froid ; at contraire, l'été de I 964 aux caractéristiques opposées nous a permis d'intéressantes comparaisons.

C'est en ayant présente à l'esprit cette notion d'efficacité pollinisatrice que nous allons passer en revue les principales espèces rencontrées.

\section{TABI,EAU I}

I.iste des pollinisateturs rencontrés sur les l'gumineuses fourragères à Lusignan (Vienne)

L'abondance relative des espèces est symbolisée de la manière suivante : fréquent + , abondant ++ , très abondant +++ . l'our des espèces moins communes, les chiffres des captures ont été donnés à titre indicatif.

Familles

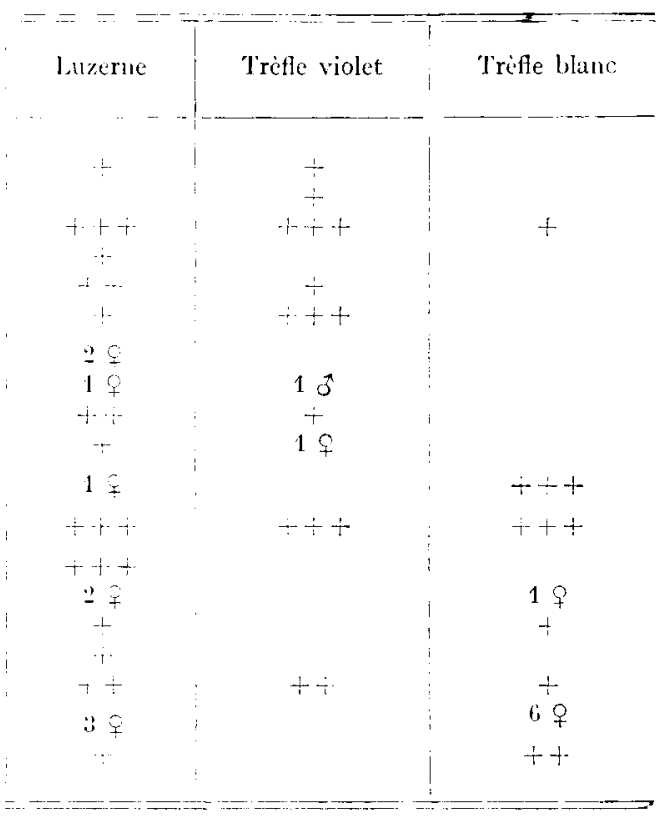

\section{Melitta leporina $\mathrm{P} \%$ :}

Cette espèce est très particulièrement abondante à Lusignan; nous l'arons d'ailleurs rencontrée dans toutes les régions visitées : Beauce, Sud-Ouest. En I964, à Lusignan, les premiers mâles furent capturés le $\mathrm{I} 7$ juin sur la L,uzerne et ils devinrent très vite innombrables ; la première femelle apparut quinze jours plus tard. Vingt jours après la première sortie, c'est-à-dire aux environs dı 20 juillet, les Melitta femelles étaient très abondantes sur les fleurs de Luzerne et de Trèfle violet et il était possible d'observer deux à quatre femelles par plante.

L,es sorties de terre des femelles sont très étagées dans le temps et il était possible de capturer des femelles fraîchement nées à la fin de juillet et au début du mois d'août. En conséquence, il est encore possible d'en rencontrer très tardivement dans la saison.

Melitta leporina est une abeille très active qui déclenche près de roo $\mathrm{p}$ Ioo des fleurs de Luzerne visitées et y récolte du pollen. Cependant les mâles qui ne visitent les 


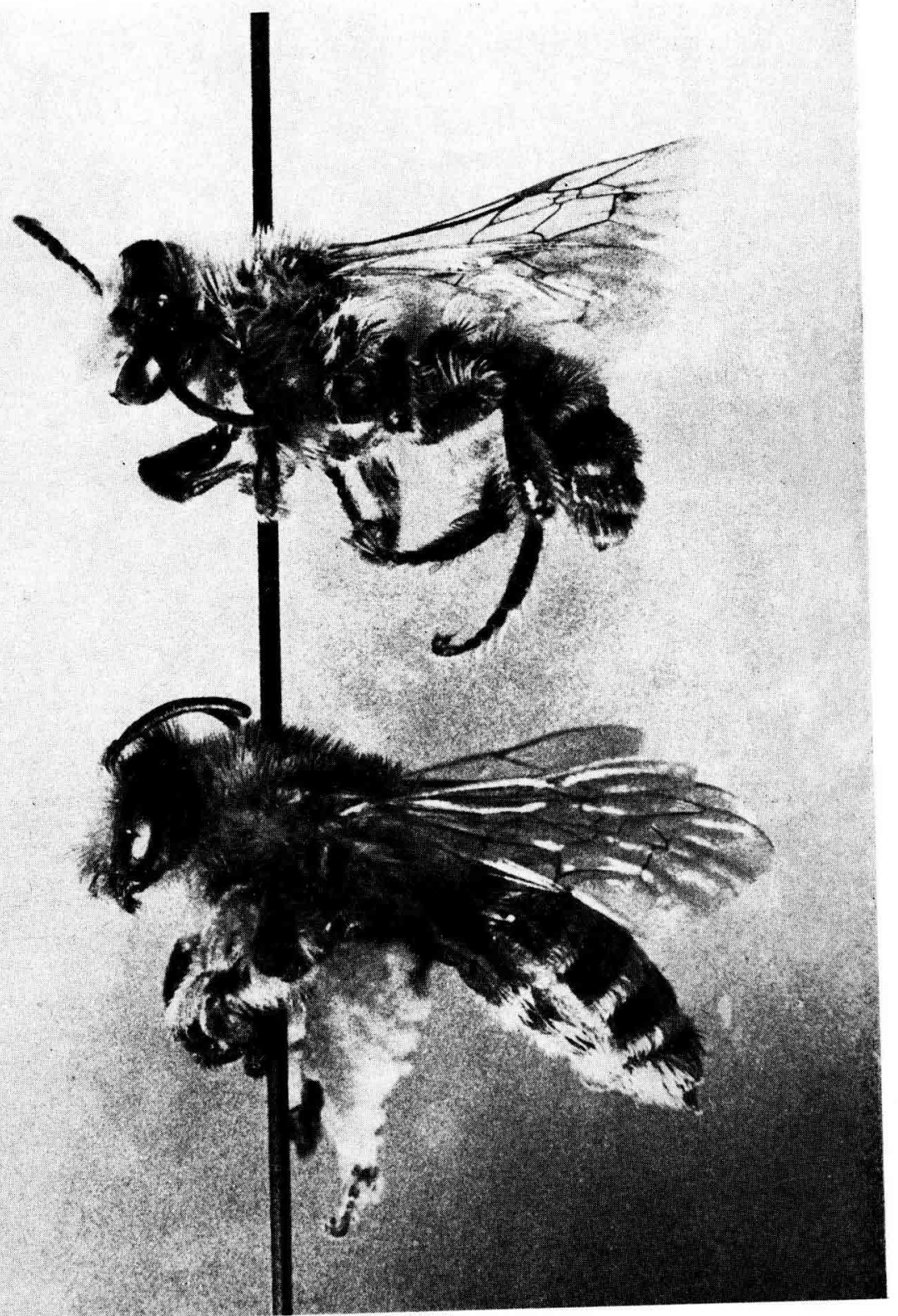

Frg. I. - Melitta leporina, mâle el jemelle. La jemelle porte des pelotes de pollen 
fleurs que pour y récolter du nectar, s'approchent des nectaires latéralement en évitant de faire fonctionner le mécanisme de déclenchement. Ce comportement est, rappelons-le, adopté par la grande majorité des ouvrières d'abeilles domestiques et constitue la raison majeure du peu d'intérêt présenté par cet insecte pour assurer une pollinisation intensive de la Luzerne.

Melitta leporina visite également très volontiers le Trèfle violet pour y récolter du pollen. Cette plante est même nettement préférée à la Luzerne quand les deux se trouvent en compétition. A la fin de juillet, ou au mois d'aôtit, quand les autres ressources deviennent rares, on observe que les Melitta se tournent vers le Trè̀le blanc, encore abondant dans les prairies, et qu'elles y récoltent également du pollen.

Les qualités de butineuses de Melitta leporina méritent d'être soulignées. Cet insecte se rencontre dès $7 \mathrm{~h}$. 30 le matin si les conditions sont favorables et il est encore présent au crépuscule. Par temps très nuageux ou quand la vitesse du vent éloigne l'abeille domestique, on rencontre encore les Melitta et il est aussi possible de les voir travailler sous la pluie, de nombreuses observations en font foi.

La nidification de Melitta leporina a lieu dans le sol et les nids sont très difficiles à trouver car, d'une part, ils ne forment pas d'agrégation, d'autre part, il semble qu'ils doivent être dissimulés par la végétation. I'un des rares nids observé était situé dans les chaumes d'un champ de blé récemment coupé à quelques mètres d'une parcelle de Luzerne.

\section{Melitturga clavicornis I.ATR.}

Le premier mâle fut également capturé le I7 juin r 964 sur des fleurs de Luzerne et neuf jours après plusieurs femelles étaient rencontrées, déjà occupées à récolter du pollen. En I963, pour des raisons qui nous sont inconnues et en dépit des conditions météorologiques très défavorables, les sorties de Melitturga avaient eu lieu un mois plus tôt.

Ces insectes apparaissent en masse, dans un assez court laps de temps et vivent environ I mois. Durant ce temps, ils sont très actifs sur les fleurs de Luzerne, qu'ils semblent préférer par dessus tout, les champs de Trèfle blanc ou violet en pleine floraison se trouvant au voisinage ne les attirant absolument pas. Les deux sexes sont capables d'opérer le déclenchement de la fleur de l,uzerne, mais seule évidemment la femelle récolte du pollen. L'un de nous (Tirgari, I965) a eu l'occasion d'étudier un site de nidification à Sierre (Stuisse), mais il nous a été impossible de découvrir ceux de la région de Lusignan. En tout état de cause, il s'agit d'une abeille portée à nidifier en bourgades importantes dans des couches plus ou moins sableuses de terre arable.

Melitturga clavicornis est une espèce méridionale que nous avons rencontrée dans tout le Sud-Ouest, plus ou moins abondante, mais que nous n'avons pas trouvée dans la région parisienne. Lusignan n'est sans doute pas très éloigné de la limite septentrionale de cet insecte.

Bombus spp.

Au cours de nos prospections, nous avons toujours été frappés par l'abondance relative des Bourdons dans les populations d'Apoïdes présentes sur les légumineuses fourragères. Au mois de mai 1964 , les reines étaient très nombreuses à Lusignan 


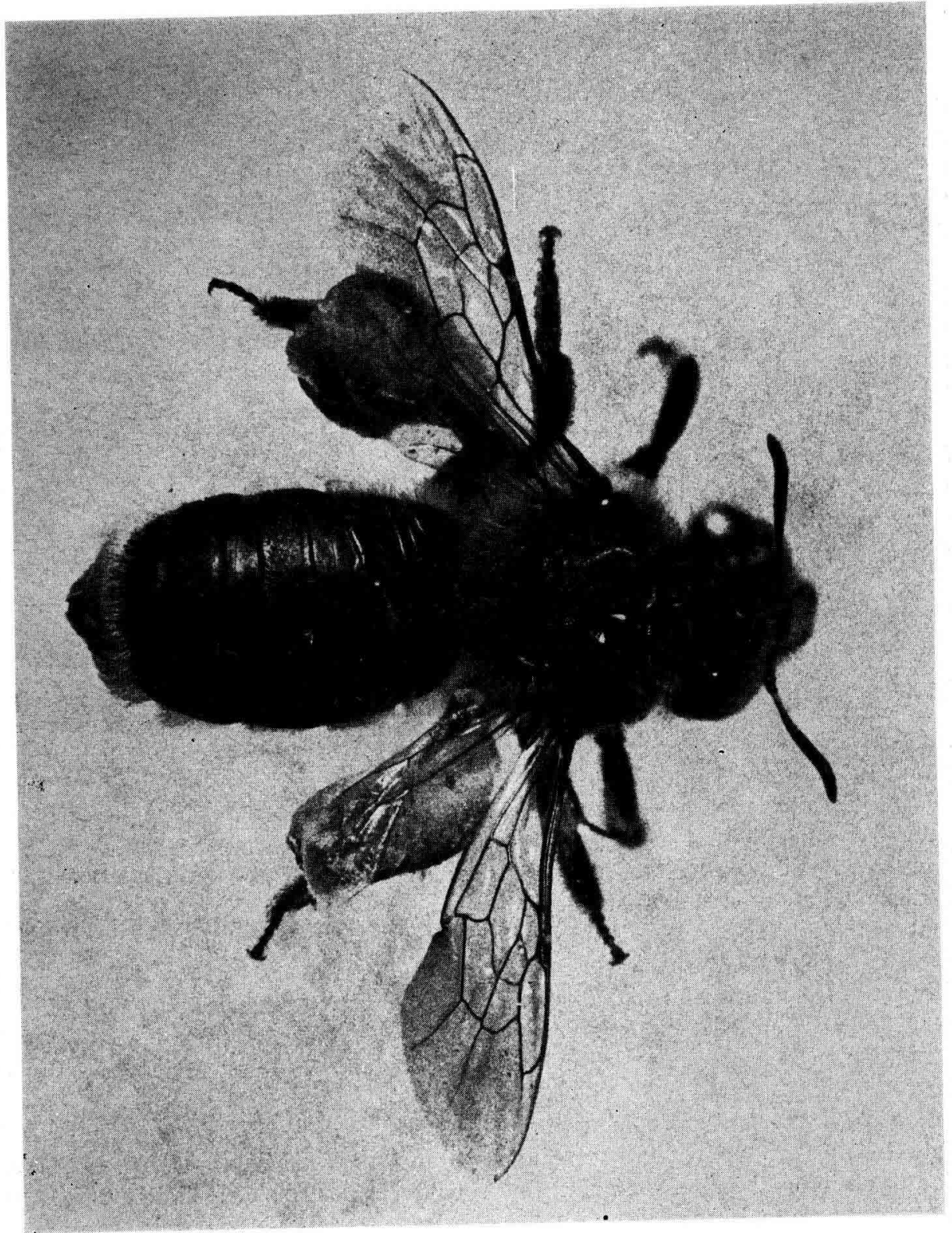

FIG. 2. - Melitturga clavicornis, fenelle portant des pelotes de pollen 


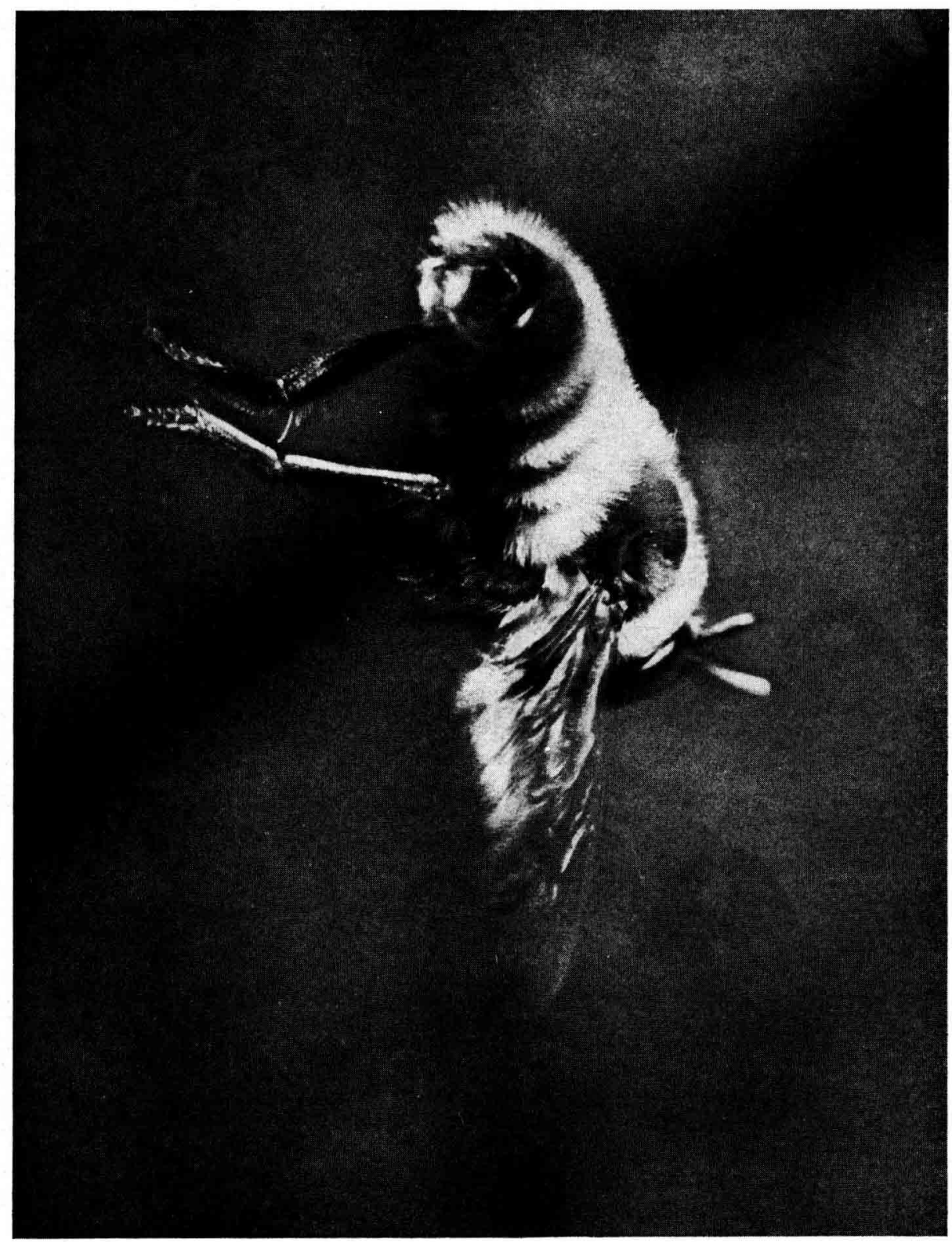

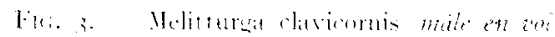


et au moment de la floraison du Trèfle violet un grand nombre d'ouvrières de différentes espèces étaient au travail. I,es populations étaient cependant bien plus nombreuses à la fin du printemps et de l'été qu'au milieu de l'été. Au mois d'août, un très grand nombre de jeunes reines et de mâles s'observaient sur les Luzernes et les Trèfles pourtant déjà très défleuris.

Les espèces dominantes en 1963 et 1964 étaient $B$. lapidarius et $B$. terrestris.

\section{Eucera longicornis $\mathrm{L}$.}

Cette abeille est connue depuis longtemps comme étant un pollinisateur de la Luzerne en Europe centrale et orientale. Cet insecte apparait de bonne heure au milieu du printemps et se rencontre encore à la fin de juillet; il visite également volontiers le 'Trèfle violet. Cependant, leur population n'est pas très considérable dans la région de Lusignan. E. longicornis se rencontre également dans la région parisienne et dans tout le Sud-Ouest, mais nous n'avons jamais observé de pullulations permettant de lui assigner un rôle primordial dans la pollinisation. Il nous a été possible d'observer la nidification d'une femelle au pied d'une touffe d'herbe à la lisière d'un champ mixte de I,uzeme et de Trèłle violet.

\section{Eucera interupta.}

Esst encore moins fréquente.

Andrena ovatula $\mathrm{K}$.

Il s'agit d'une abeille relativement précoce que l'on rencontre sur le 'Trèfle violet et la Luzerne. Fille est particulièrement abondante à Lusignan au milieu du printemps et des sorties se manifestent tout au long de l'été

Il nous a été possible de capturer des femelles fraîchement écloses durant la période normale de floraison de la Luzerne cultivée pour la production grainière. Filles semblent avoir une préférence marquée pour le Trèfle violet et ceci est particulièrement frappant quand on considère les populations présentes sur une parcelle de Trèfle violet et sur un vaste champ de Luzerne adjacent. Cependant, ces insectes visitent volontiers la Luzerne s'il n'y a pas compétition.

$A$. ovatula est une abeille solitaire qui nidifie dans le sol. En Ig6́3, nous avons pu observer 8 femelles nidifiant dans un espace d'environ deux mètres carrés à la base même de touffes d'herbe, dans une prairie ombragée par des arbres.

Les femelles semblent d'excellents pollinisateurs de la Luzerne quand elles visitent cette plante; les mâles, qui sont beaucoup plus petits, récoltent du nectar sans actionner le mécanisme de déclenchement.

Halictus xanthopus кву.

Se rencontre très souvent à L,usignan sur le Trèfle blanc où il récolte đu nectar, bien qu'il soit souvent porteur de pollen et puisse jouer un rôle pollinisateur il ne récolte cependant pas le pollen du 'Trèfle blanc. Nous l'avons observé en 1963 récoltant du pollen de Coquelicot (Papaver rheas).

Une remarque assez analogue peut être faite en ce qui concerne Halictus longulus SM (malachurus K.) qui se rencontre en abondance dans certaines parties de la Vendée sur les fleurs de Luzerne. Cet insecte ne semble pas capable de déclencher les fleurs et ne porte du pollen que pour avoir visité des fleurs déclenchées par un 
autre insecte. Les fleurs visitées pour obtenir normalement le pollen sont des Composées, d'ailleurs généralement abondantes dans les champs de Luzerne eux-mêmes. Dans ces conditions, le rôle pollinisateur est bien difficile à apprécier. Il n'en sera pas de même avec Rophites canus qui est connu comme un excellent pollinisateur de la Luzerne, ce que nous avons pu vérifier. Malheureusement, cette abeille qui était très abondante en fin de juillet et début août I960 dans la région d'Auneau (Eureet-Loir) s'est considérablement raréfiée depuis et n'a pas été rencontrée dans d'autres localités. Il s'agit aussi d'une espèce nidifiant en bourgades et, de ce fait, très intéressante, mais nous n'avons pas pu localiser leurs emplacements.

\section{TABLEAU 2}

Évolution des populations des principales espèces d'insectes pollinisateurs présentes sur les fleurs des légumineuses fourragères en 1964 dans la région de Lusignan (Vienne). L'épaisseur de la figure est proportionnelle à l'importance de la population.

\begin{tabular}{|c|c|c|c|c|c|c|}
\hline espèces & Avril & Mai & Juin & Juillet & Aout & Septembre \\
\hline \multicolumn{7}{|l|}{$\begin{array}{l}\text { Melitta } \\
\text { leporina }\end{array}$} \\
\hline \multicolumn{7}{|l|}{$\begin{array}{l}\text { Melitturga } \\
\text { clavicornis }\end{array}$} \\
\hline \multicolumn{7}{|l|}{ Bombus spp. } \\
\hline \multicolumn{7}{|l|}{$\begin{array}{l}\text { Andrena } \\
\text { ovatula }\end{array}$} \\
\hline $\begin{array}{l}\text { Eucera } \\
\text { longicornis }\end{array}$ & & & & & & \\
\hline
\end{tabular}

Nous avons résumé dans le tableau 2 un certain nombre d'informations concernant les populations des principaux pollinisateurs observés en I964 dans la région de Lusignan.

\section{CONCLUSIONS}

L'inventaire des pollinisateurs autres que l'abeille domestique que nous avons effectué nous a montré que, parmi la vingtaine d'espèces rencontrées, les plus abondantes étaient Melitta leporina, Melitturga clavicornis, Andrena ovatula, Eucera longicornis, Bombus lapidarius et $B$. terrestris. $M$. leporina peut être considérée comme le principal pollinisateur des trois légumineuses fourragères, dans la région de Lusignan. Dans le cas de la Y,uzerne, elle partage cependant ce rôle avec $M$. clavicornis qui, au moins en I964, a joué un rôle plus important. Les autres espèces 
dominantes ne jouent peut-être pas, considérées individuellement, un rôle majeur mais leur ensemble est certainement responsable d'un pourcentage important de la pollinisation.

De toute manière, l'existence d'un éventail assez large d'espèces pollinisatrices représente un avantage certain, en ce sens qu'elle évite les situations catastrophiques qui peuvent survenir du fait de la réduction inopinée et momentanée d'une espèce ou d'un décalage des sorties dans le temps.

Enfin, il est possible de faire certaines remarques au sujet des mesures à prendre dans l'état actuel de nos connaissances pour continuer à pouvoir profiter d'une population naturelle de pollinisateurs.

I $^{0}$ Assurer la mise à fleur des plantes au moment oì le plus grand nombre de pollinisateurs se trouvent en activité, c'est-à-dire les trois dernières semaines de juillet en ce qui concerne la Luzerne dans la région de Lusignan.

$2^{\circ}$ Éliminer, si possible, les plantes pouvant être compétitrices, nous avons vu que le Trèfle violet détournait Melitta leporina de la Luzerne.

$3^{\circ}$ Éviter les traitements insecticides au moment de la floraison ou aux approches de la floraison dans le cas de produits très rémanents.

$4^{\circ}$ Préserver les sites de nidification, surtout lorsqu'il s'agit d'espèces nidifiant en bourgades. Ces mesures de protection seront évidemment plus faciles à appliquer quand nous aurons amélioré notre connaissance des habitudes de nidification. Il semble, en tout état de cause, que les prairies âgées soient particulièrement précieuses à cet égard.

Reçu pour publication en avril 1965.

\section{REMERCIEMEN'TS}

Il nous est particulièrement agréable de remercier ceux qui nous ont aidé dans la détermination des insectes étudiés, en particulier le Dr L. Moczar du Muséum d'histoire naturelle de Budapest, le $\mathrm{D}^{\mathrm{r}} \mathrm{G}$. Bohart de l'C niversité de Iogan (U.S. A.), le pr Delmas de l'École nationale supérieure agronomique de Montpellier, Mlle Kelner-Pillaut du Muséum d'histoire naturelle de Paris, Mme Plateau-(juent du laboratoire d'Évolution à Paris. Nous tenons également à remercier M. DEMARLy, Directeur et le persomnel de la Station d'Amélioration des P'lantes fourragères de Lusignan pour l'aide apportée au cours de ce travail.

\section{SUMMARY}

ON SOME POLLINISATORS OF THE FODDER LEGUMES

The authors have drawn a list of the main pollinisators of fodder legumes in the Paris area, the West and South-West of France. Among the twenty species which have been noted, the most important ones seem to be Melitta leporina and Melliturga clavicornis.

This paper includes a certain number of indications on the biology of some species as well as comments on the measures which will enable us to go on making use of the natural populations of pollinisators, in the places where they exist. 


\section{RÉFÉRENCES BIBLIOGRAPHIQUES}

ARxason A. P., 1946. Recent studics in Canada of crop pollination by insects. IInd Intem. Simposium on Pollination, I,ondon.

Bischofe H., 1927. Biologie der Iymenopteren. Berlin, Julius Springer, 186-188, 227-228.

Bohart G. E., 1957. Pollination of alfalfa and red clover. Ann. Rev. Entomol., 2, 355-380.

Bohart G. E., 1958. Alfalfa pollinators with special reference to species other than honey bees. Yl Intenn. Cong. Entomol., 4, 929-937.

BoharT G. E., 1960. Insect pollination of forages legumes. Bee World, 4, 57-6.4-85-97.

Chauvir R., 1952. Études d'écologic entomologiques sur le champ de Luzernc. .1nn. de l'I. N. R. A., LXI-I,XXXII.

Chacvis R., ig6o. La faune du chanp cultivé et surtout du champ de Luzerne, revue des travaux récents. Rev. 'oool. agric. appl., 59, 14-37;69-79; 102-109; 1 38-151.

ENslix E., I92r. Beiträge zur kenntnis der Hymenopteren (Rhophites canus Evers. and Discoelius zonalis PAxz.). Dtsch. Entomol. Z., 11, 59-64.

Lecomte J., 1959. Premières observations sur le comportement des insectes pollinisateurs de la Luzerne. Ann. Abeille, 4, 277-84.

I.ecomte J., I962. Techniques d'étude des populations d'insectes pollinisateurs. Anu. Abeille, 5, $201-2$ г 3 . Linsi.ey E. G., 1964. Insect pollinators of alfalfa in California. J. Econ. Entonol, 36, 18-26.

Miyamoto S., 1962. Outline of flower relationships of Japanese bees. . Acta Hymenopterologica, 1, $393^{-455}$. Moczar L., 196t. The distribution of wild bees in the lucerne fields of Hungary (Hymenoptera, Apoidea). Ann. Hist. nal. Mus. Vil. Hung., 53, 45I-46r.

Popov V. P., 1958. Bees, their relations to melittophilous plants and the problem of alfalfa pollination, $\mathrm{X}$ Intern. Cong. Entomol., 4, 983-990.

TIRGARI S., 1965. Observation sur un site de nidification de Melilurga clanicornis latr. (En cours de publication).

ToDD E. E., 1957. Insect pollination of legumes. Grass land seeds, W. A. Wheeler et D. D. Hill, Princeton. N. J., Van Tostrand. 